\title{
Whole-body low-dose computed tomography in multiple myeloma staging: Superior diagnostic performance in the detection of bone lesions, vertebral compression fractures, rib fractures and extraskeletal findings compared to radiography with similar radiation exposure
}

\author{
LUKAS LAMBERT $^{1}$, PETR OUREDNICEK ${ }^{2}$, ZUZANA MECKOVA $^{3}$, \\ GIAMPAOLO GAVELLI ${ }^{4}$, JAN STRAUB $^{5}$ and IVAN SPICKA ${ }^{5}$ \\ ${ }^{1}$ Department of Radiology, First Faculty of Medicine, Charles University and General University Hospital in Prague, \\ 12808 Prague; ${ }^{2}$ Department of Imaging Methods, St. Anne's University Hospital in Brno, 65691 Brno; \\ ${ }^{3}$ Institute of Nuclear Medicine, First Faculty of Medicine, Charles University and General University Hospital in Prague, \\ 12808 Prague, Czech Republic; ${ }^{4}$ Radiology Unit, IRCCS-Istituto Scientifico Romagnolo per lo Studio e la Cura dei \\ Tumori (IRST), I-47014 Meldola, Italy; ${ }^{5}$ Department of Hematology, First Faculty of Medicine, \\ Charles University and General University Hospital in Prague, 12808 Prague, Czech Republic
}

Received July 14, 2016; Accepted November 17, 2016

DOI: $10.3892 / \mathrm{ol} .2017 .5723$

\begin{abstract}
The primary objective of the present prospective study was to compare the diagnostic performance of conventional radiography (CR) and whole-body low-dose computed tomography (WBLDCT) with a comparable radiation dose reconstructed using hybrid iterative reconstruction technique, in terms of the detection of bone lesions, skeletal fractures, vertebral compressions and extraskeletal findings. The secondary objective was to evaluate lesion attenuation in relation to its size. A total of 74 patients underwent same-day skeletal survey by CR and WBLDCT. In CR and WBLDCT, two readers assessed the number of osteolytic lesions at each region and stage according to the International Myeloma Working Group (IMWG) criteria. A single reader additionally assessed extraskeletal findings and their significance, the number of vertebral compressions and bone fractures. The radiation exposure was $2.7 \pm 0.9 \mathrm{mSv}$ for WBLDCT and $2.5 \pm 0.9 \mathrm{mSv}$ for $\mathrm{CR}(\mathrm{P}=0.054)$. $\mathrm{CR}$ detected bone involvement in 127 out of 486 regions $(26 \%$; $\mathrm{P}<0.0001)$, confirmed by WBLDCT. CR underestimated the disease stage in $16 \%$ and overestimated it in $8 \%$ of the patients $(\mathrm{P}=0.0077)$. WBLDCT
\end{abstract}

Correspondence to: Dr Lukas Lambert, Department of Radiology, First Faculty of Medicine, Charles University and General University Hospital in Prague, U Nemocnice 2, 12808 Prague, Czech Republic E-mail: lambert.lukas@gmail.com

Key words: plasma cell disorder, staging, osteolytic lesion, computed tomography, x-ray detected more rib fractures compared with CR (188 vs. 47; $\mathrm{P}<0.0001$ ), vertebral compressions (93 vs. $67 ; \mathrm{P}=0.010$ ) and extraskeletal findings (194 vs. 52; $\mathrm{P}<0.0001)$. There was no correlation observed between lesion size $(\geq 5 \mathrm{~mm})$ and its attenuation $(\mathrm{r}=-0.006 ; \mathrm{P}=0.93)$. The inter-observer agreement for the presence of osteolytic lesions was $\kappa=0.76$ for WBLDCT, and $\kappa=0.55$ for CR. The present study concluded that WBLDCT with hybrid iterative reconstruction technique demonstrates superiority to $\mathrm{CR}$ with an identical radiation dose in the detection of bone lesions, skeletal fractures, vertebral compressions and extraskeletal findings, which results in up- or downstaging in $24 \%$ patients according to the IMWG criteria. The attenuation of osteolytic lesions can be measured with the avoidance of the partial volume effect.

\section{Introduction}

Clonal plasma cell disorders comprise a set of hematological conditions with substantial differences not only in laboratory parameters, such as hypercalcemia, renal insufficiency and anemia, bone marrow findings, including infiltration by clonal plasma cells, and patient complaints and prognosis, but also in the therapeutic approach $(1,2)$. Imaging has an integral role in the decision making process of whether to initiate treatment (3). The presence and number of osteolytic lesions detected by radiographic skeletal survey was one of the key criteria in the original 1975 Durie-Salmon staging system (4), which was later revised in the context of expanding cross-sectional imaging (5). Currently, the evaluation of skeletal findings is based on the International Myeloma Working Group (IMWG) criteria for monoclonal gammopathies and multiple myeloma, which distinguishes between the presence or absence of structural changes, regardless of their quantity $(3,6)$. 
The superiority of cross-sectional imaging methods including whole body computed tomography (CT), magnetic resonance imaging (MRI) or positron emission tomography (PET) in detecting osteolytic lesions (resulting in up- or downstaging of disease) in individual patients has previously been established in several studies (6-9). However, knowledge remains limited concerning quantitative comparison of region-based detection mismatch between conventional radiography (CR) and whole-body low-dose CT (WBLDCT), with similar radiation exposure using hybrid iterative reconstruction technique, inter-observer agreement, as well as the evaluation of skeletal fractures, vertebral compressions and extraskeletal pathology, and the utility of lesion characterization by its attenuation.

The primary objective of the present study was to compare skeletal survey by the means of CR and WBLDCT, with similar radiation exposure reconstructed with hybrid iterative reconstruction technique in terms of the number of involved regions, region-based mismatch, staging, skeletal fractures and vertebral compressions, as well as extraskeletal findings. The secondary objective of the present study was to evaluate image quality and lesion attenuation in relation to its size to establish whether a reliable measurement of its attenuation can be performed.

\section{Materials and methods}

Patient information. The present prospective study was performed in accordance with the Declaration of Helsinki and was approved by the Ethics Committee of the General University Hospital (Prague, Czech Republic). All patients signed informed consent. A total of 74 patients underwent skeletal survey using CR and WBLDCT on the same day, between May 2013 and May 2015. The mean age of the patients was $65.1 \pm 10.7$ years, $53 \%$ were males and their body mass index was $26.5 \pm 3.5 \mathrm{~kg} / \mathrm{m}^{2}$. Patients had the following types of myeloma $(2,3)$ : Immunoglobulin $(\operatorname{IgG}) \kappa, 43 \% ; \operatorname{IgG} \lambda$, $22 \%$; $\operatorname{IgA} \lambda, 12 \%$; IgA $\kappa, 7 \%$; serum free light chains (sFLC) $\kappa, 7 \%$; sFLC $\lambda, 4 \%$; IgG $\kappa+\lambda, 1 \%$; cFLC $\lambda, 1 \%$; non-secretory, $1 \%$; and $\operatorname{IgM}, 1 \%$. A total of 54,15 and $31 \%$ of patients were classified as International Staging System stage I, II and III, respectively (10). In total, $50 \%$ of the patients had been previously treated by chemotherapy and/or stem cell transplant.

$C R$. Radiographs were acquired on a clinical computed radiography system (Multix Pro; Siemens AG, Munich, Germany) with standard settings for each of the following 19 projections: Skull [anteroposterior (AP), lateral (LL)], cervical, thoracic and lumbar spine (AP, LL), chest [posterioanterior (PA)], ribs (AP), pelvis (AP), thigh (AP), leg (AP), arm (AP) and forearm (AP). The images were reviewed on a clinical workstation (Fujitsu-Siemens Esprimo P5925; Siemens AG) with medical grade display (NEC MD213MG; NEC Display Solutions Europe GmbH, Munich, Germany) and clinical viewer (Syngo Imaging XS, version VA70B; Siemens AG).

WBLDCT. Unenhanced WBLDCT scans were performed on a 256-slice scanner (Brilliance iCT 256; Philips Healthcare, Best, The Netherlands) with the following parameters: Peak tube voltage, $120 \mathrm{kV}$; planned tube time-current product,
$30 \mathrm{mAs}$; detector collimation, $128 \times 0.625 \mathrm{~mm}$; rotation time, $0.5 \mathrm{sec}$; pitch, 0.993 ; matrix size, $512 \times 512$; reconstructed in $0.9 \mathrm{~mm}$ sections using filter $\mathrm{C}$ (medium sharp reconstruction kernel) and hybrid iterative reconstruction technique $\left(\right.$ iDose $^{4}$ ) set on level 4 (of 6). The scans were reviewed on a multimodality client workstation (Intellispace Portal v4.0.4; Philips Healthcare) by two independent readers experienced in reading skeletal radiographs and CT (10 and 20 years' experience, respectively).

Assessment of CR and WBLDCT results. In CR and WBLDCT, the readers reported the number of osteolytic lesions in every region ( 1 to 5 ; or $>5$ ) and stage according to the current IMWG criteria (3). The first reader also assessed extraskeletal findings and their clinical importance (unimportant; likely unimportant; potentially important finding) and the number of vertebral compressions and skeletal fractures (11). The first reader additionally measured the size of the largest lesion in every region and, in lesions $\geq 5 \mathrm{~mm}$, their attenuation (3). The quality of WBLDCT examination was rated on a four-point Likert scale (0, excellent; 1 , standard; 2 , suboptimal; 3 , insufficient) (12).

Calculation of the radiation dose. In ten randomly chosen patients, the radiation dose from radiography was calculated using PCXMC version 2.0 (STUK, Helsinki, Finland), and from WBLDCT using ImPACT CT Patient Dosimetry Calculator 1.0.4 (ImPACT, London, UK). In the remaining patients, a calculated weighting factor of $0.093 \mathrm{mSvmGy}{ }^{-1} \mathrm{~cm}^{-1}$ was used to estimate the radiation dose from WBLDCT using the dose length product (DLP) (13).

Statistical analysis. Statistical tests were performed using MedCalc, version 12 (MedCalc Software, Ostend, Belgium). To test for statistical significance, the Wilcoxon signed rank test or pairwise $t$-tests were used for comparison between WBLDCT and CR. Correlation between the attenuation and size of osteolytic lesions was expressed as Cohen's $\kappa . P<0.05$ was considered to indicate a statistically significant difference.

\section{Results}

CR detected bone involvement in 127 out of 486 regions (26\%; $\mathrm{P}<0.0001$ ), confirmed by WBLDCT. CR underestimated disease stage according to the IMWG criteria in $16 \%$ and overestimated it in $8 \%$ of the patients $(\mathrm{P}=0.0077$; Fig. 1A). Region-based comparison between CR and WBLDCT is shown in Figs. 2 and 3. The inter-observer agreement for the presence of osteolytic lesions was $\kappa=0.76[95 \%$ confidence interval (CI), 0.72-0.80] for WBLDCT, and $\kappa=0.55$ (95\% CI, 0.50-0.62) for CR (Fig. 4). WBLDCT detected more vertebral compressions ( 93 vs. $67 ; \mathrm{P}=0.010$ ), rib fractures (188 vs. 47 ; $\mathrm{P}<0.0001)$ and skeletal fractures elsewhere ( 15 vs. $7 ; \mathrm{P}=0.006$; Fig. 1B) compared with CR. The mean size of the lesions missed by CR was $9.3 \mathrm{~mm}$ [interquartile range (IQR) $4.3-17.2 \mathrm{~mm}$ ], compared with the mean size of lesions detected by $\mathrm{CR}$, which was $12.2 \mathrm{~mm}$ (IQR 4.5-22.2 mm) $(\mathrm{P}=0.29$; Fig. 1C). There was no correlation between size of the lesions $(14.5 \mathrm{~mm}$; IQR, 8.4-23 mm) and their attenuation (18.5 Hounsfield units; IQR, -13-49; Spearman's r=-0.006; $\mathrm{P}=0.93)$. 
A

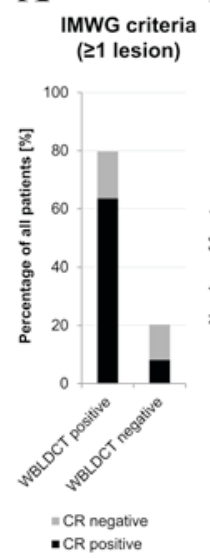

B

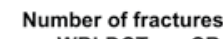
on WBLDCT vs. CR

C Size of lesions missed

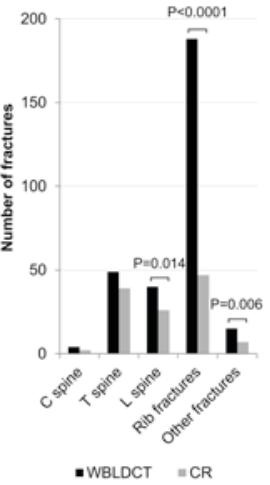
on $\mathrm{CR}$

50

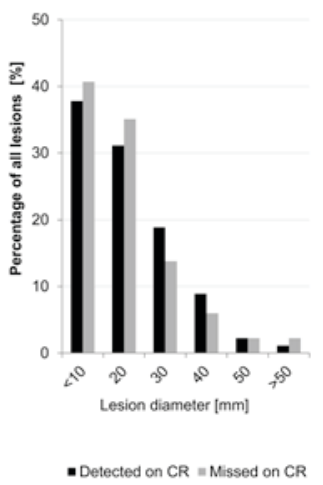

Figure 1. (A) Compared to WBLDCT, CR underestimated the disease stage in $16 \%$ and overestimated it in $8 \%$ of the patients. (B) WBLDCT detected more vertebral compressions overall ( 93 vs. $67 ; \mathrm{P}=0.010)$, rib fractures (188 vs. $47 ; \mathrm{P}<0.0001)$ and skeletal fractures elsewhere $(15$ vs. $7 ; \mathrm{P}=0.006)$. (C) The mean size of skeletal lesions missed by CR was $9.3 \mathrm{~mm}$ compared to the lesions detected by $\mathrm{CR}$ with $12.2 \mathrm{~mm}(\mathrm{P}=0.29)$. IMWG, International Myeloma Working Group; WBLDCT, whole-body low-dose computed tomography; $\mathrm{CR}$, conventional radiography; $\mathrm{C}$ spine, cervical spine; $\mathrm{T}$ spine, thoracic spine; L spine, lumbar spine.

\section{Comparison of the number of involved regions between WBLDCT and CR}

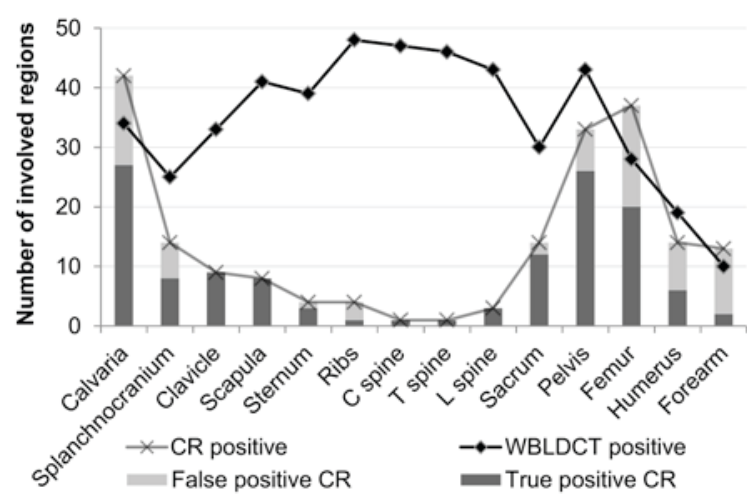

Figure 2. Comparison of the number of regions involved by osteolytic bone lesions between CR and WBLDCT (74 patients). CR, conventional radiography; WBLDCT, whole-body low-dose computed tomography; C spine, cervical spine; T spine, thoracic spine; L spine, lumbar spine.

In total, there were 52 secondary findings detected on $\mathrm{CR}$, $60 \%$ of them as a result of previous surgery or interventional procedure. None of these were deemed clinically significant. CT showed 194 extraskeletal findings $(\mathrm{P}<0.0001) ; 16 \%$ of them were procedure-associated and an additional $16 \%$ were evaluated as being clinically significant or requiring further investigation. They included the presence of a soft tissue mass, lymphadenopathy, aneurysm of abdominal aorta, pneumonia, ascites, non-cystic liver lesions, pulmonary mass or nodule (>10 mm), splenomegaly, hepatomegaly, complicated renal cyst and hemopericardium.

The mean DLP was $289 \pm 85 \mathrm{mGy} \cdot \mathrm{cm}^{-1}$ resulting in a radiation dose estimate of $2.7 \pm 0.9 \mathrm{mSv}$ on CT. The radiation dose estimate from radiographic examinations was $2.5 \pm 0.9 \mathrm{mSv}$ $(\mathrm{P}=0.054)$. The score for WBLDCT image quality rated on 4-point Likert scale was $1.1 \pm 0.3$.

\section{Sensitivity and specificity of CR in the detection of bone lesions compared to WBLDCT}

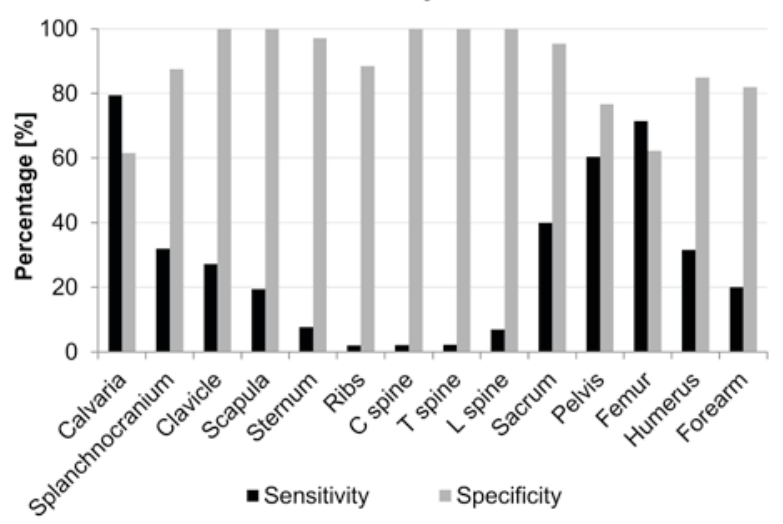

Figure 3. Sensitivity and specificity of CR in the detection of osteolytic bone lesions compared to WBLDCT, grouped by regions. CR, conventional radiography; WBLDCT, whole-body low-dose computed tomography; C spine, cervical spine; $T$ spine, thoracic spine; $L$ spine, lumbar spine.

\section{Interobserver agreement}

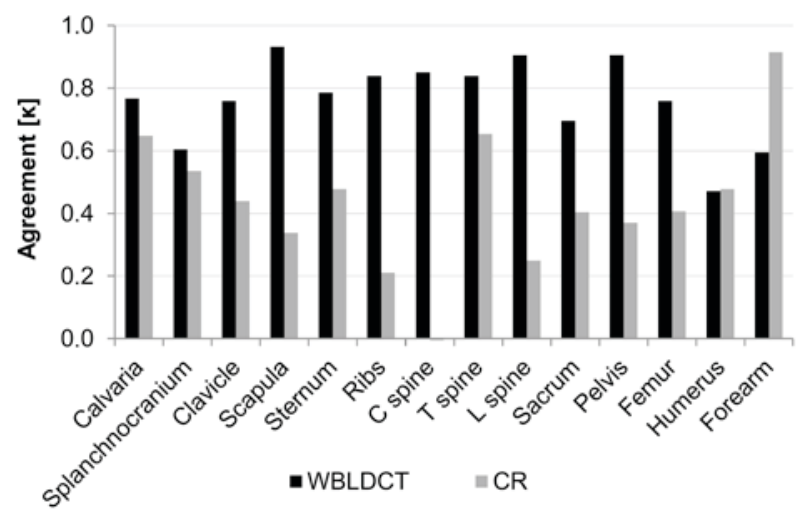

Figure 4. Inter-observer agreement in the detection of osteolytic bone lesions in each region expressed as kappa $(\kappa)$ ranged from moderate to almost perfect in WBLDCT and from no agreement to almost perfect agreement in CR. In general, the inter-observer agreement for the presence of osteolytic lesions was $\kappa=0.76$ (substantial agreement) for WBLDCT, and $\kappa=0.55$ (moderate agreement) for CR. WBLDCT, whole-body low-dose computed tomography; $\mathrm{CR}$, conventional radiography; $\mathrm{C}$ spine, cervical spine; $\mathrm{T}$ spine, thoracic spine; L spine, lumbar spine.

\section{Discussion}

Skeletal survey by CR is a basic method for the detection of lytic lesions in the staging of clonal plasma cell disorders (4), which has been observed to be outperformed by CT, MRI and PET-CT (3).

Several varying protocols for whole body $\mathrm{CT}$ in the staging of multiple myeloma have been reported. Standard or low-dose protocols with either 120 or $100 \mathrm{kV}$ peak tube voltage and 40-100 mAs planned tube time-current have been used without iterative reconstruction technique, with a resulting radiation exposure between 4 and $10 \mathrm{mSv}(7,14)$. Gleeson et al (15) utilized a $140 \mathrm{kV}$ protocol with $14 \mathrm{mAs}$ or $25 \mathrm{mAs}$, and an average radiation exposure of $3.3 \mathrm{mSv}$, which resulted in adequate image quality in $82 \%$ of patients. Although $140 \mathrm{kV}$ peak tube voltage results in improved penetration of the $\mathrm{X}$-rays compared to $120 \mathrm{kV}$, it also decreases contrast between 


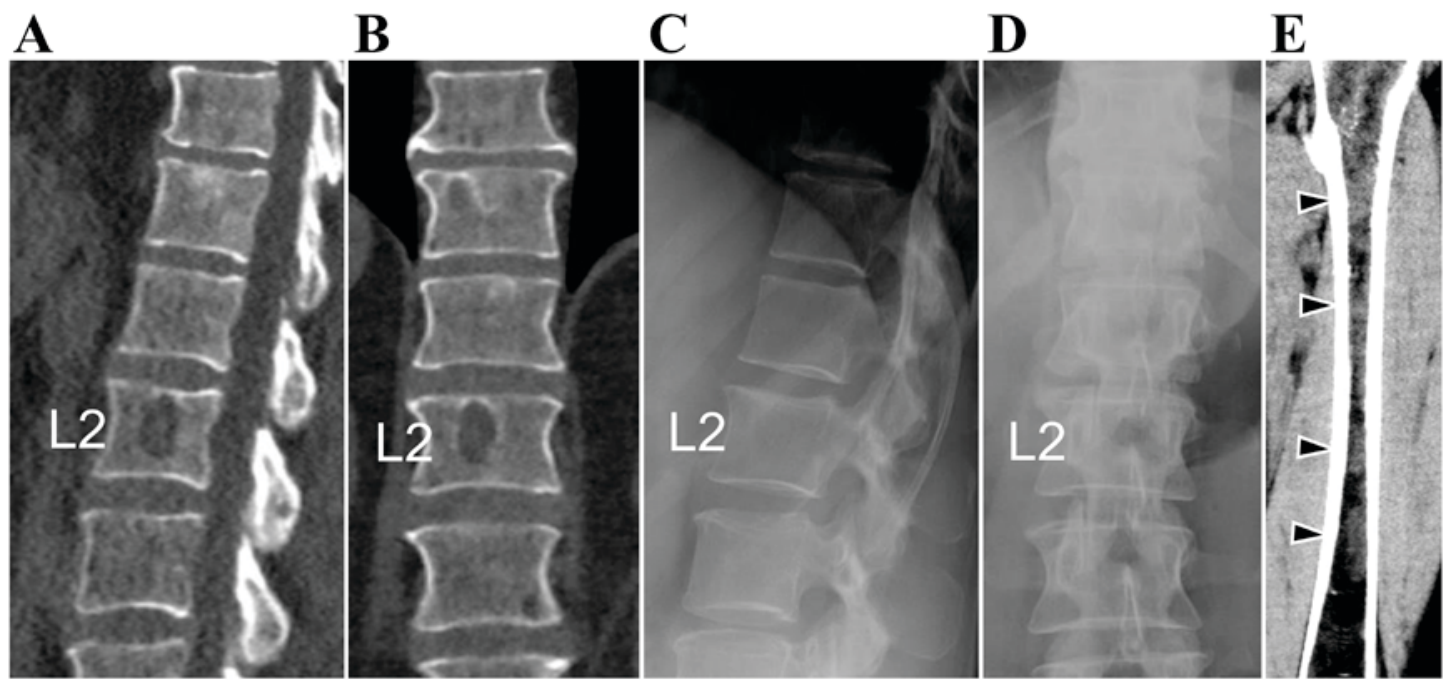

Figure 5. Comparison of conspicuity of a $18 \mathrm{~mm}$ osteolytic lesion in the second lumbar vertebra (L2) between WBLDCT images in the (A) sagittal plane and (B) coronal plane and conventional radiography in the (C) lateral view and (D) anterior-posterior view. Even large lesions are difficult to diagnose with certainty by radiography due to superimposition with other structures that form a heterogeneous background. In (B) the coronal plane, image artifacts are less conspicuous than in (A) the sagittal plane. (E) WBLDCT also reveals islands of bone marrow infiltration in the femur (black arrowheads). L2, second lumbar vertebra; WBLDCT, whole-body low-dose computed tomography.

bone calcium and soft tissue and increases the radiation exposure (16). As recent advances in iterative reconstruction technique offer significant improvements in image quality and a reduction of image noise and artifacts compared to standard filtered projection, the radiation dose may be decreased even further without sacrificing the image quality, as proposed in the present study.

For the radiation dose and resulting image quality, positioning of the patient has an important role (17). If the torso is scanned with arms placed alongside the body, a 30\% greater radiation dose is required to compensate for the attenuation caused by the arms. Fortunately certain artifacts, including beam hardening and ring artifacts (most prominent in the lumbar spine), may be mitigated by positioning the arms more anteriorly (17). This results in the shifting of the 'ring' artifacts in the same direction, thus improving the image quality of the spine. Furthermore, the present study observed that these artifacts are less appreciated if the spine is viewed in the coronal rather than the sagittal plane, as is commonly done (Fig. 5).

The present study confirmed that compared to WBLDCT, $\mathrm{CR}$ is able to identify less than one-third of regions involved by osteolytic lesions $(9,17)$. Additionally, the present study demonstrated that the poorest diagnostic performance of $\mathrm{CR}$ could be expected in the spine and rib cage. Large bony destruction of a vertebral body must occur before it becomes detectable by CR, by which time the vertebral body would have mostly collapsed (Fig. 5). Changes in bone structure due to porosis or degenerative disease are better distinguished from osteolytic lesions on $\mathrm{CT}$, even though demineralization decreases perceived image quality on low dose scans (15).

In terms of the therapeutic impact, rather than the precise number of lesions or the affected regions, it is more important to quantify the change of the stage (6). As the majority of patients undergoing skeletal survey exhibit multiple lesions, the lack of sensitivity of CR, particularly in the spine, is mitigated by imaging of other involved regions, particularly the appendicular skeleton, calvaria and pelvis.
The present study demonstrated that there was no correlation between the lesion size and its attenuation, indicating that its attenuation can be measured with the avoidance of partial volume effect. This is a significant finding because myeloma lesions change their attenuation with treatment (18). Similar changes that occur in the bone marrow of the long bones may also be detected not only by MRI, but also by CT $(19,20)$. Further study is warranted to research whether this feature may be used to select patients who have entered remission.

Secondary findings (unrelated to the indication of the examination) are an integral part of imaging. The present study confirmed that CR grossly underestimates extraskeletal findings, including those requiring further investigation or treatment.

The present study has several limitations. Firstly, a single CT scanner was used, but it was considered that solutions by other major vendors with hybrid or model based iterative reconstruction technology would be comparable. Furthermore, the radiation dose from CR examination varies considerably, even within a single institution. In addition, due to ethical considerations, the present study did not include healthy controls and there were only $20 \%$ of patients without osteolytic lesions. Finally, the study group was inhomogeneous, and half of the patients had previously undergone treatment.

In conclusion, WBLDCT skeletal survey with hybrid iterative reconstruction technique demonstrates superiority to $\mathrm{CR}$ with a comparable radiation dose in the detection of bone lesions, skeletal fractures, vertebral compressions and extraskeletal findings, which results in up- or downstaging in $24 \%$ of the patients, according to the IMWG criteria. CR has poorer performance in the detection of osteolytic lesions, particularly in the spine and rib cage. The attenuation of osteolytic lesions may be measured with avoidance of the partial volume effect, but additional study is required to demonstrate whether its changes could be used as an auxiliary biomarker of therapeutic response. 


\section{Acknowledgements}

The present study was supported by the First Faculty of Medicine of Charles University (Prague, Czech Republic; grant no., PRVOUK-P27/LF1/1) and by the Ministry of Health of the Czech Republic (Prague, Czech Republic; grant no. RVO VFN 64 165).

\section{References}

1. Bird JM, Owen RG, D'Sa S, Snowden JA, Pratt G, Ashcroft J, Yong K, Cook G, Feyler S, Davies F, et al: Guidelines for the diagnosis and management of multiple myeloma 2011. Br J Haematol 154: 32-75, 2011.

2. Rajkumar SV: Myeloma today: Disease definitions and treatment advances. Am J Hematol 91: 90-100, 2016.

3. Rajkumar SV, Dimopoulos MA, Palumbo A, Blade J, Merlini G, Mateos MV, Kumar S, Hillengass J, Kastritis E, Richardson $\mathrm{P}$, et al: International Myeloma Working Group updated criteria for the diagnosis of multiple myeloma. Lancet Oncol 15: e538-e548, 2014.

4. Durie BG and Salmon SE: A clinical staging system for multiple myeloma. Correlation of measured myeloma cell mass with presenting clinical features, response to treatment, and survival. Cancer 36: 842-854, 1975.

5. Durie BG, Kyle RA, Belch A, Bensinger W, Blade J, Boccadoro M, Child JA, Comenzo R, Djulbegovic B, Fantl D, et al: Myeloma management guidelines: A consensus report from the Scientific Advisors of the International Myeloma Foundation. Hematol J 4 : 379-398, 2003

6. Dimopoulos M, Terpos E, Comenzo RL, Tosi P, Beksac M, Sezer O, Siegel D, Lokhorst H, Kumar S, Rajkumar SV, et al International myeloma working group consensus statement and guidelines regarding the current role of imaging techniques in the diagnosis and monitoring of multiple Myeloma. Leukemia 23: 1545-1556, 2009

7. Kröpil P, Fenk R, Fritz L, Blondin D, Kobbe G, Mödder U and Cohnen M: Comparison of whole-body 64-slice multidetector computed tomography and conventional radiography in staging of multiple myeloma. Eur Radiol 18: 51-58, 2008.

8. Horger M, Kanz L, Denecke B, Vonthein R, Pereira P, Claussen CD and Driessen C: The benefit of using whole-body, low-dose, nonenhanced, multidetector computed tomography for follow-up and therapy response monitoring in patients with multiple myeloma. Cancer 109: 1617-1626, 2007.

9. Princewill K, Kyere S, Awan O and Mulligan M: Multiple myeloma lesion detection with whole body CT versus radiographic skeletal survey. Cancer Invest 31: 206-211, 2013.
10. Greipp PR, San Miguel J, Durie BG, Crowley JJ, Barlogie B, Bladé J, Boccadoro M, Child JA, Avet-Loiseau H, Kyle RA, et al: International staging system for multiple myeloma. J Clin Oncol 23: 3412-3420, 2005.

11. Zalis ME, Barish MA, Choi JR, Dachman AH, Fenlon HM, Ferrucci JT, Glick SN, Laghi A, Macari M, McFarland EG, et al: CT colonography reporting and data system: A consensus proposal. Radiology 236: 3-9, 2005.

12. Allen BC, Baker ME, Einstein DM, Remer EM, Herts BR, Achkar JP, Davros WJ, Novak E and Obuchowski NA: Effect of altering automatic exposure control settings and quality reference $\mathrm{mAs}$ on radiation dose, image quality, and diagnostic efficacy in MDCT enterography of active inflammatory Crohn's disease. Am J Roentgenol 195: 89-100, 2010.

13. Huda W, Magill D and He W: CT effective dose per dose length product using ICRP 103 weighting factors. Med Phys 38: 1261-1265, 2011

14. Horger M, Claussen CD, Bross-Bach U, Vonthein R, Trabold T, Heuschmid $\mathrm{M}$ and Pfannenberg C: Whole-body low-dose multidetector row-CT in the diagnosis of multiple myeloma: An alternative to conventional radiography. Eur J Radiol 54: 289-297, 2005.

15. Gleeson TG, Moriarty J, Shortt CP, Gleeson JP, Fitzpatrick P, Byrne B, McHugh J, O'Connell M, O'Gorman P and Eustace SJ: Accuracy of whole-body low-dose multidetector CT (WBLDCT) versus skeletal survey in the detection of myelomatous lesions and correlation of disease distribution with whole-body MRI (WBMRI). Skeletal Radiol 38: 225-236, 2009.

16. Anderson NG, Butler AP, Scott NJ, Cook NJ, Butzer JS, Schleich N, Firsching M, Grasset R, de Ruiter N, Campbell M and Butler PH: Spectroscopic (multi-energy) CT distinguishes iodine and barium contrast material in MICE. Eur Radiol 20: 2126-2134, 2010.

17. Downey K, Castellano E, Mears D, Kaiser M and Messiou C: Low-dose whole-body CT for staging multiple myeloma. Clin Radiol 70 (Suppl 1): S7, 2015.

18. Schulze M, Weisel K, Grandjean C, Oehrlein K, Zago M, Spira D and Horger M: Increasing bone sclerosis during bortezomib therapy in multiple myeloma patients: Results of a reduced-dose whole-body MDCT study. AJR Am J Roentgenol 202: 170-179, 2014.

19. Martínez-Martínez F, Kybic J, Lambert L and Mecková Z: Fully automated classification of bone marrow infiltration in low-dose CT of patients with multiple myeloma based on probabilistic density model and supervised learning. Comput Biol Med 71: 57-66, 2016.

20. Nishida Y, Matsue Y, Suehara Y, Fukumoto K, Fujisawa M, Takeuchi M, Ouchi E and Matsue K: Clinical and prognostic significance of bone marrow abnormalities in the appendicular skeleton detected by low-dose whole-body multidetector computed tomography in patients with multiple myeloma. Blood Cancer J 5: e329, 2015. 